\title{
Identity Management in Internet of Things: A Software Defined Networking Approach
}

\author{
Kazi Masum Sadique ${ }^{1}$, Rahim Rahmani ${ }^{1}$, Paul Johannesson ${ }^{1}$ \\ ${ }^{1}$ Department of Computer and Systems Sciences, Stockholm University, \\ Borgarfjordsgatan 8, SE-164 07 Kista, Sweden \\ \{sadique, rahim,paul\}@dsv.su.se
}

\begin{abstract}
Internet of things (IoT) network needs to accommodate billions of connected smart objects (things). Connected IoT objects should be able to communicate with each other. Objects should be able to travel between different networks regardless of their locations, network providers, and manufacturers. To allow transparent movement of IoT objects it is very important to have a trust relationship between these objects. To establish trusted relationship, unique identity is one of the key properties for any IoT object. It will not be possible for the devices to freely move within the networks, if we do not have a common identity solution. The use of software defined networking (SDN) approach in IoT is increasing these days, due to its flexibility and easy adaptability with any network. In this paper we have presented an SDN based identity management system which will possibly solve the unique identity problem and increase trust in heterogeneous IoT network.
\end{abstract}

Keywords: Internet of things, Identity management, Security, Trust, and Software defined network.

\section{Introduction}

The quality of human life is improving everyday with the vast implementation of smart Internet of Things (IoT) objects in everything. The application of IoT technology includes smart health, smart cities, smart farming, smart energy, smart home, smart vehicle, smart waste management and many more [1]. IoT technology introduces networks of anything. To achieve network of anything, heterogeneous and interconnection capabilities between smart objects is crucial. It means that the IoT objects should be able to be connect or disconnect to any network that it could belongs to, regardless of time, location and its' hardware manufacturer [2]. One of the common use cases of IoT objects/nodes are to act as sensors or actuators. These sensors and actuators are typically communicating with a local gateway. The gateways connect to a centralized server to share date produced from the end nodes. The centralized servers are located on the cloud. A centralized control from a cloud platform for globally distributed IoT nodes is becoming difficult in respect to security, when billions of nodes need to transfer data to and from a centralized server. Real time authentication and authorization of these smart nodes from a centralize server is 
also challenging [3]. At many critical situations, waiting for response from a centralized cloud server could be very harmful [4]. These lead to the concept of decentralized management of IoT nodes instead of controlling from a cloud server.

\section{Background and Motivations}

Mobility capabilities of wireless sensor nodes open new opportunities and use cases for smart healthcare, smart agriculture, cattle tracking, smart traffic management, vehicle location tracking, battlefield surveillance and so on [16]. Management of bullions of dynamic smart nodes from central server is not a best solution for the future IoT. There are also security issues such as node authentication, integrity and confidentiality of data, key management, identity management which need to be addressed. Previous research shows that a public/private key pair based approach is appropriate for resource constraint sensor nodes [17]. We have discussed authentication and identity management below before describing our proposed solution.

\subsection{Authentication}

Authentication is process of ensuring a valid entity within any system. It is a kind of service that concerned with assuring that relation within premises is authentic. Authentication is the first step to perform any computing operation to go to the next step. To be granted access to the system usually entity needs to provide some kinds of secret or piece of information known by the privileged entity. Identification is crucial for authentication [5].

\subsection{Identity Management}

Previous researches show that identity management is one of the issues that need further research [5]. Future IoT infrastructure will combine billions of smart objects with flexible movement. All these smart objects carry context information that can allow further track within the network. To allow flexible movement of smart objects in the IoT networks, unique identity of devices is very crucial. Also to ensure authentication and authorization devices need unique identity.

Considering the future IoT infrastructure scenario and above described security issues, we found that unique identity management solution is essential for future IoT paradigms. A question can be arrived that why do we need a distributed solution and not a centralized control? The answers are

- Billions of smart nodes need to be able to connect to the network dynamically without any human assistance.

- Without any difficulty future IoT nodes should be able to move between different gateways regardless of location. 
- Single point of failure at a centralize solution will possibly interrupt the system, even if we keep redundant standby servers.

- Management of identity of billions of nodes from a centralized server located at cloud is not easy and not an efficient solution.

- Time critical decision making is not possible using a centralized solution.

\section{Related work}

There are many recent papers addressed the software defined networking (SDN) based IoT security model and architectures, but as per our knowledge none of those have proposed a decentralized identity management architecture. Mike et al. has proposed SDN-IoT architecture with network function virtualization (NFV) implementation to address challenges in IoT. The paper also addressed business aspects of using SDN and NFV based IoT architecture [6]. Oliver et al. pointed out that a combination of SDN and OpenFlow based software switches affect the performance of IoT devices. The proposed SDN model was designed to secure both wired and wireless network communication including Ad-Hoc network objects like mobile phones, sensor, tablets, or household apparels [7]. Sahoo et al. highlighted the security challenges in IoT and proposed an SDN based network architecture. They have also shown in a comparison table with several security issues such as unauthorized access, data leakage, data modification, malicious application, denial of service, configuration issue exists among them the authors find out that control and data layers are most vulnerable. There are two major reasons are found out throughout this research are single point failure and coordination among multiple controllers. To prevent attacks grid of security was introduced even though a large portion of issues still needs to take care of [8]. Ding et al. discussed that fast networking comes with lots of opportunities as well as unwanted security issues that lead to a security breach. So, their focus in the paper is it possible to overcome wireless network security issues using SDN. The key elements of the authors' solution framework include local agents, central controller, and security layer for wireless mobile networks. According to the authors' observation, there is a lack of concern for SDN security in the wireless and mobile domain. The OpenFlow API and its north bound interfaces already provide a good foundation for innovative solutions on top of the existing SDN framework, especially for security elements [9].

\section{Distributed SDN based identity management}

In our previous work [10], we suggested architecture for future IoT network where we described identity management based on context information. In our proposed architecture, we have considered location and time information to produce unique identity of any smart object in IoT network. We have designed a location-based identity provider server for IoT network. There are few basic assumptions: we have assumed that the smart objects are the end nodes which are involved in different specific task as per requirement of the application. The nodes are always transmitting 
or receive data via an edge device (a gateway). We didn't consider distributed software defined networking approach. The magic of SDN based solution is layered separation of control and data plane. This makes SDN solution efficient and easy maintainable [11]. We realized that our previously proposed solution needs extension. This paper is a significantly modified presentation of our previous work. The fig. 1 presents the network architecture of our previously proposed context-based identity solution. Identity management is done using identity provider server. Every nodes and gateways with a specific location get their unique identity from the identity provider server. When a node moves to a new location it needs to confirm its own identity before performing any kind of data transfer to and from a gateway. The identity provider server at the new location confirms the identity of the new node with the identity server at the previous location.

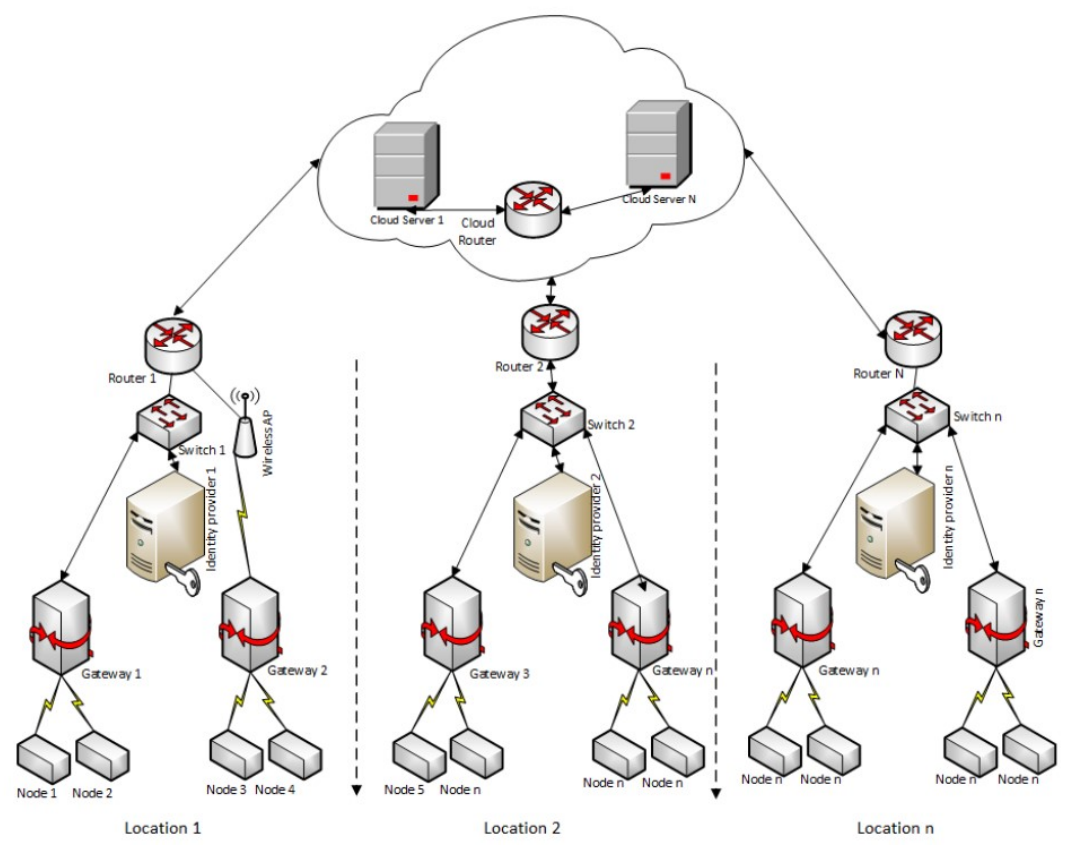

Fig. 1. Architecture of location based identity management system [10]

A distributed identity management architecture shown in Fig. 1, it can be an example scenario for future $5 \mathrm{G}$ network where all the edge devices connect to the cloud storage via $5 \mathrm{G}$ network and the unique identities of those devices are assigned by the software defined identity management system. The problem with the above proposed solution is single point of failure and also communication between different identity servers can be time consuming and we also found that with a traditional presentation of identity server with routers and switches is not a robust solution. Based on our analysis we have modified our previously proposed architecture with distributed software defined network solution. In our proposed solution in Fig. 2, we have 
suggested distributed solution with sync capabilities. Local nodes and gateways share parameters with the local SDN server to get identity. The SDN device verifies the request and assigns a unique identity to the node or gateway. In Fig. 2 we have considered nodes and gateways as node only. To ensure reliability, the local SDN device syncs its identity database with the Global SDN device. All Global SDN devices also sync their databases.

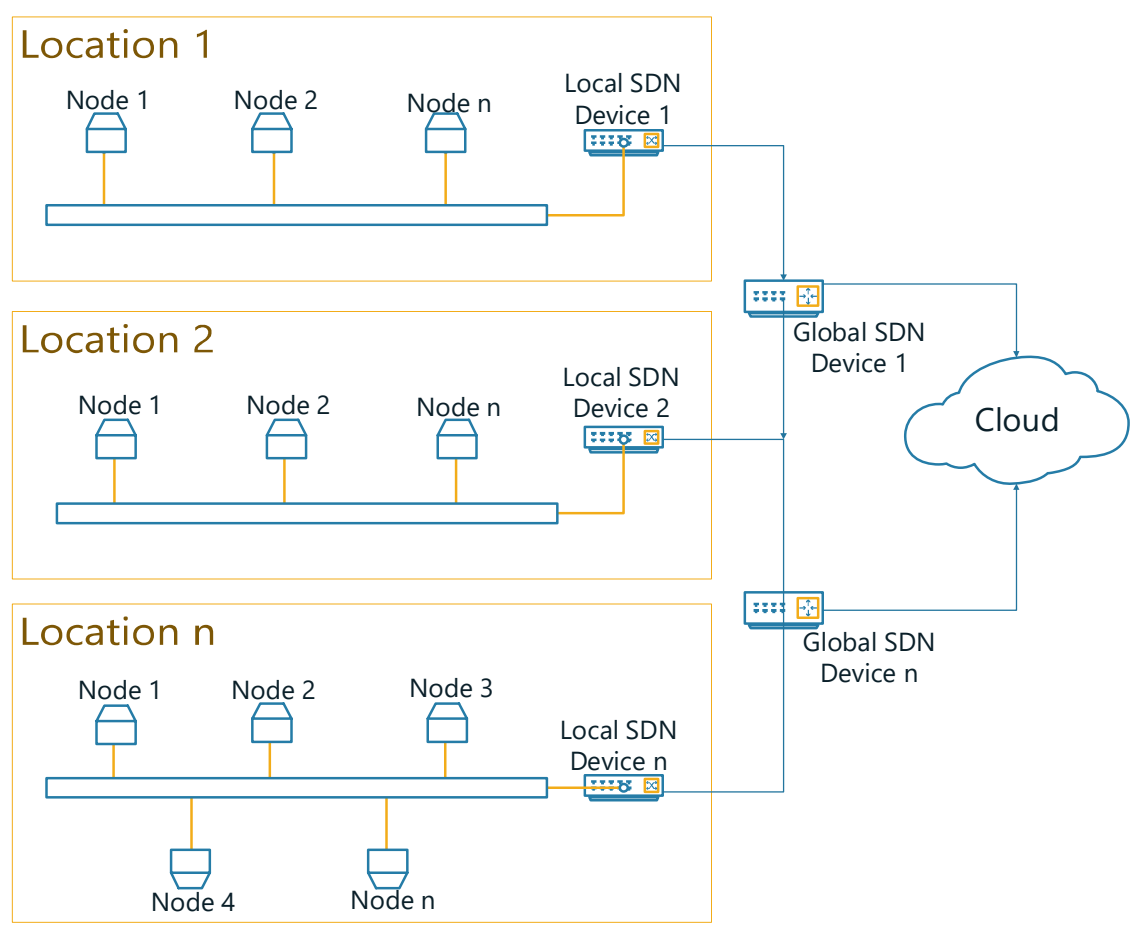

Fig. 2. Architecture of distributed SDN based identity management system

To understand the operation of our designed identity provider we have to consider few scenarios. An example scenario for context based moving entities is vehicle network. Moving vehicles needs to collaborate and share information between each other which we can define as vehicle's cooperation as a cluster [13]. In a multivehicle infrastructure, nodes can make decisions based on context at two levels in response to a) the unavailability of communications paths and b) application level events and context changes [14]. Finally, we require interworking between the multivehicles, which in essence is a peer-to-peer IoT infrastructure [15] and a cloud-based IoT platform hosted in a data-center. 


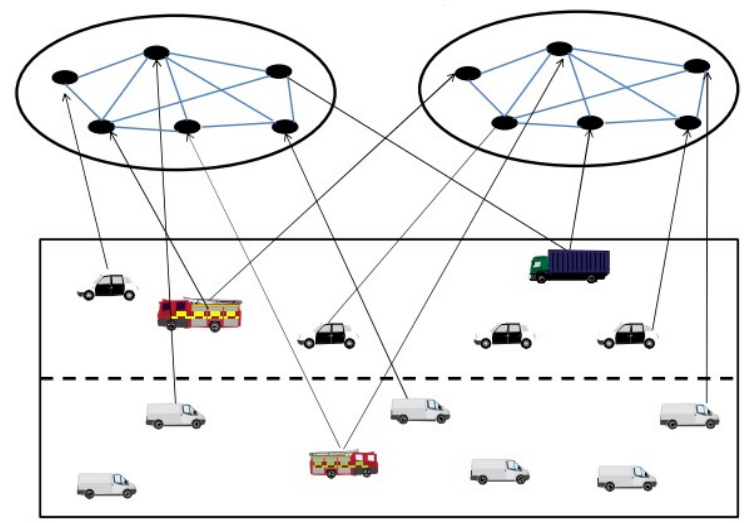

Fig. 3. Example scenario of a context aware vehicular network diagram [12]

Scenarios 1: A newly connected smart node needs to transmit data to the gateway. The join to a gateway is confirmed in few steps. We have described the steps below:

- $\quad$ Node makes a RequestToJoin to the nearest gateway

- Gateway replies with a RequestToGetIdentity from the local SDN device

- Gateway sends information about the RequestToJoin from a node to the local SDN device

- Node makes RequestToGetIdentity to the local SDN device

- Local SDN device verifies the requests received from the node and gateway and assigns a unique identity to the node and shares IdentityAssigned with the node and the gateway

Messages with context information for the above scenario:

RequestToJoin: A newly installed smart object sends request to join to the nearest gateway. The object includes own hardware address, current location and own public key in a packet.

RequstToGetIdentity: When the gateway gets a request to join, it checks if the object trying to join has a unique identity information or not. If no identity information found at the gateway database, it sends a RequstToGetIdentity message with the identity and public key of the local SDN device.

IdentityAssigned: When a unique identity is assigned by a local SDN device, it shares this information to the respective gateway and the requesting node.

RequestToSync: This message is send when a local SDN device shares update of unique identity table with the global SDN device.

Scenarios 2: A node moved to a new location and needs to join a new gateway. The join to a gateway is confirmed in few steps. We have described the steps below: 
- Node makes a RequestToJoin to the gateway at the new location with the unique identity received from the local SDN device at the previous location

- Gateway checks if there is any identity information exist for this node in its' database, if not gateway check for the identity of the node in the local SDN device

- If Local SDN device can't find an entity at its' local database, it communicates with the SDN device located at the previous location of the device

- Based on the reply from the SDN device located at the previous location, local SDN server replies to the gateway about the identity of the node

- Gateway confirms or denies the RequestToJoin request from the node based on the identity verification result from the local SDN device

We have proposed the following algorithm for the SDN based identity management device when it assigns a unique identity to an end node:

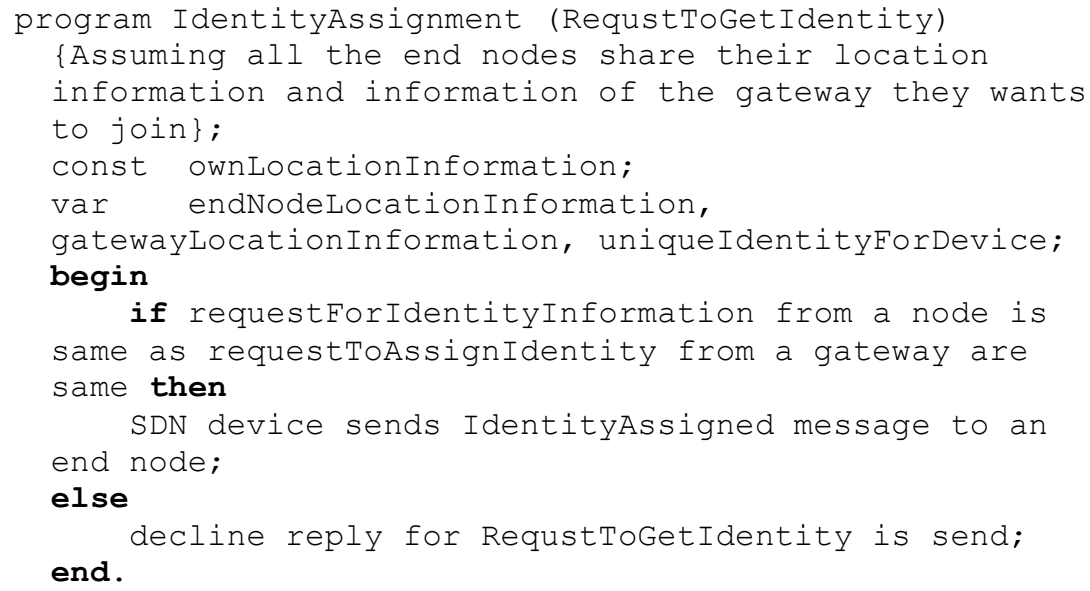

The below algorithm is for synchronization of identity information between local and global identity devices.

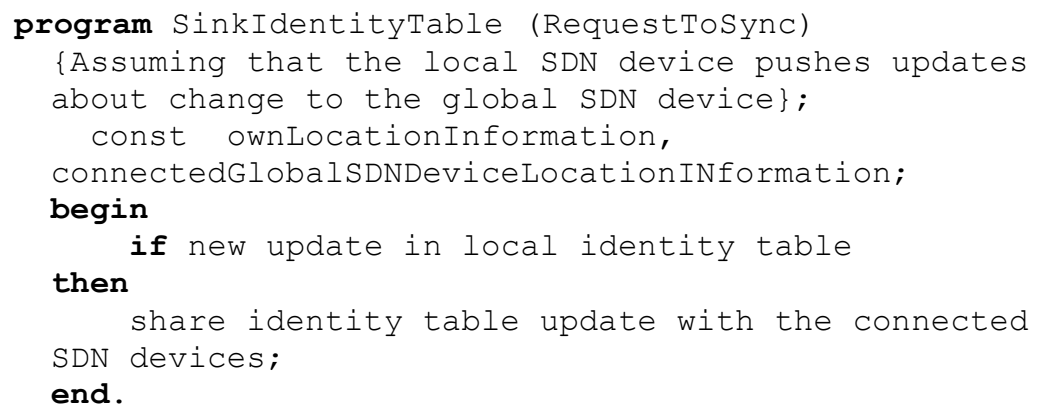


In Table 1, we have presented different variable parameters for different devices which will be connected in the proposed architecture to share and update identity information of different devices.

Table 1. Parameters for the proposed algorithms.

\begin{tabular}{ll}
\hline Device & Parameter \\
\hline End node & $\begin{array}{l}\text { Own current location information, Own previous } \\
\text { location information, own key pair (public and } \\
\text { private), own identity information, current gateway } \\
\text { identity information, current gateway public key, } \\
\text { previous gateway identity information, software } \\
\text { defined identity provider's identity and public key }\end{array}$ \\
Gateway device & $\begin{array}{l}\text { Own location information, own key pair (public and } \\
\text { private), own identity information, connected nodes } \\
\text { identity information public key and location }\end{array}$ \\
& $\begin{array}{l}\text { information, software defined identity provider's } \\
\text { identity and public key }\end{array}$ \\
Local software defined & $\begin{array}{l}\text { Own location information, own key pair (public and } \\
\text { private), own identity information, identity } \\
\text { identity provider } \\
\text { certain location, identity information of the global }\end{array}$ \\
Global software defined & $\begin{array}{l}\text { Own location information, own key pair (public and } \\
\text { private), own identity information, identity } \\
\text { identity provider } \\
\text { information of end nodes and gateways within } \\
\text { different location (synced by the local SDN device), } \\
\text { identity information of the local software defined } \\
\text { identity provider devices }\end{array}$ \\
\hline
\end{tabular}

\section{Discussion and future work}

We have proposed a fault-tolerant aggregation algorithm based on distributed software defined networking. This software defined identity management solution will have the following benefits:

- The proposed architecture has the possibility to solve unique identity problems of billions of IoT devices.

- The problem of single point of failure will be reduced by using this distributed software defined architecture.

- The system should be easy manageable and configurable.

- Controller update and upgrade process will be very easy without any interruption of the authentication process.

Our fault-tolerant approach is proactive and based on a novel design of a future architecture for authentication and identity management of IoT nodes and gateways. 
Although the proposed approach distributes trust and noise for different privacy among the meters we showed that it can gracefully tolerate single node fail due to communication failures.

\section{References}

1. Talari, S., Shafie-Khah, M., Siano, P., Loia, V., Tommasetti, A., \& Catalão, J.: A review of smart cities based on the internet of things concept. Energies, 10(4), (2017) 421

2. Patel, K. K., \& Patel, S. M.: Internet of things-IOT: definition, characteristics, architecture, enabling technologies, application \& future challenges. International journal of engineering science and computing, (2016)

3. Yu, W., Liang, F., He, X., Hatcher, W. G., Lu, C., Lin, J., \& Yang, X.: A survey on the edge computing for the Internet of Things. IEEE access, 6, (2018) 6900-6919

4. Lin, J., Yu, W., Yang, X., Yang, Q., Fu, X., \& Zhao, W.: A real-time en-route route guidance decision scheme for transportation-based cyberphysical systems. IEEE Transactions on Vehicular Technology, 66(3), (2017) 2551-2566

5. Mahmoud, R., Yousuf, T., Aloul, F., \& Zualkernan, I.: Internet of things (IoT) security: Current status, challenges and prospective measures. In 2015 10th International Conference for Internet Technology and Secured Transactions (ICITST) (2015)336-341

6. Ojo, M., Adami, D., \& Giordano, S.. A SDN-IoT architecture with NFV implementation. In 2016 IEEE Globecom Workshops (GC Wkshps) (2016) 1-6

7. Olivier, F., Carlos, G., \& Florent, N.. New security architecture for IoT network. Procedia Computer Science, 52, (2015) 1028-1033.

8. Sahoo, K. S., Sahoo, B., \& Panda, A.: A secured SDN framework for IoT. In 2015 International Conference on Man and Machine Interfacing (MAMI) (2015)

9. Ding, A. Y., Crowcroft, J., Tarkoma, S., \& Flinck, H.: Software defined networking for security enhancement in wireless mobile networks. Computer Networks, 66, (2014) 94-101.

10. Sadique KM, Rahmani R, Johannesson P. Trust in Internet of Things : An architecture for the future IoT network. In International Conference on Innovation in Engineering and Technology (ICIET) (2018)

11. Jammal, M., Singh, T., Shami, A., Asal, R., \& Li, Y.: Software defined networking: State of the art and research challenges. Computer Networks, 72, (2014) 74-98

12. Kanter, T., Rahmani, R., Li, Y., \& Xiao, B.: Vehicular network enabling large-scale and real-time immersive participation. In International Conference on Internet of Vehicles (2014) 66-75.

13. Rahmani, R., Rahman, H., \& Kanter, R.: "On performance of logical-clustering of flowsensors." arXiv preprint arXiv:1401.7436 (2014).

14. Rahmani, R., Rahman, S.M.H., \& Kanter, T.: "Context-Based Logical Clustering of Flow-Sensors-Exploiting HyperFlow and Hierarchical DHTs." RNIS: Research Notes in Information and Service Sciences 14 (2013) 721-728.

15. Rahmani, R., \& Kanter, T.: "Layering the Internet-of-Things with Multicasting in Flowsensors for Internet-of-services." International Journal of Multimedia and Ubiquitous Engineering 10.12 (2015) 37-52.

16. Khan, S.U., Pastrone, C., Lavagno, L., \& Spirito, A.M.: "An energy and memory-efficient key management scheme for mobile heterogeneous sensor networks." 2011 6th International Conference on Risks and Security of Internet and Systems (CRiSIS). IEEE, 2011.

17. Seo, S-H., Won, J., Sultana, S., Bertino, E.: "Effective key management in dynamic wireless sensor networks." IEEE Transactions on Information Forensics and Security 10.2 (2015): 371-383. 